\title{
Femtosecond Laser Crystallization of Boron-doped Amorphous Hydrogenated Silicon Films
}

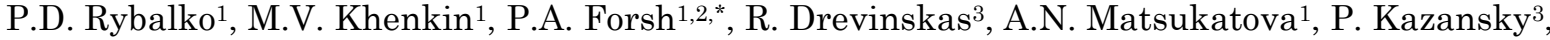 \\ A.G. Kazanskii ${ }^{1}$ \\ ${ }^{1}$ Lomonosov Moscow State University, Faculty of Physics, 119991 Moscow, Russia \\ 2 National Research Centre "Kurchatov Institute", 123182 Moscow, Russia \\ ${ }^{3}$ Optoelectronics Research Centre, University of Southampton, Southampton, SO17 1BJ, UK
}

(Received 18 June 2016; published online 03 October 2016)

\begin{abstract}
Crystallization of amorphous hydrogenated silicon films with femtosecond laser pulses is one of the promising ways to produce nanocrystalline silicon for photovoltaics. The structure of laser treated films is the most important factor determining materials' electric and photoelectric properties. In this work we investigated the effect of femtosecond laser irradiation of boron doped amorphous hydrogenated silicon films with different fluences on crystalline volume fraction and electrical properties of this material. A sharp increase of conductivity and essential decrease of activation energy of conductivity temperature dependences accompany the crystallization process. The results obtained are explained by increase of boron doping efficiency in crystalline phase of modified silicon film.
\end{abstract}

Keywords: Semiconductors, Thin films, Amorphous hydrogenated silicon, Femtosecond laser crystallization, Raman spectra, Conductivity.

DOI: 10.21272/jnep.8(3).03038

PACS numbers: 71.23.Cq, 72.80.Nq, 73.61.Jc

\section{INTRODUCTION}

Thin films of amorphous (a-Si:H) and nanocrystalline (nc-Si:H) hydrogenated silicon are used to form tandem structures for solar cell applications [1]. a-Si:H absorbs light better in short-wavelength region of the solar spectrum, while nc-Si:H is a better absorber for longer-wavelength light. Therefore, the usage of these materials in a tandem leads to the increase of the absorption of sunlight and to the increase of the efficiency.

One of the most effective manufacturing methods for nc-Si:H is a laser crystallization of a-Si:H films. Lasers with nano-, pico- and femtosecond pulse duration can be used for this purpose. Application of femtosecond lasers with light quanta energies less than the mobility edge of a-Si:H allows to realize crystallization that is uniform along the thickness of the films [2]. In the majority of published works devoted to the laser crystallization of amorphous silicon undoped amorphous silicon is used as a precursor material. At the same time there is almost no data on the properties of the modified by laser radiation doped a-Si:H. Therefore, it is of interest to conduct these researches, particularly with the boron doped films. Femtosecond laser irradiation of a-Si:H films with various laser fluences may lead to the formation of materials with essentially different structures and properties. So, the results of researches of electric and photoelectric properties of boron doped a-Si:H films that were irradiated with femtosecond laser pulses with various laser fluences are presented in this paper.

\section{EXPERIMENTAL}

a-Si:H films with a thickness of $300 \mathrm{~nm}$ were prepared by plasma enhanced chemical vapor deposition from a gas phase during decomposition of monosilane $\left(\mathrm{SiH}_{4}\right)$ and diborane $\left(\mathrm{B}_{2} \mathrm{H}_{6}\right)$ mix in plasma of the highfrequency glow-discharge. The temperature of quartz substrate was fixed to $250{ }^{\circ} \mathrm{C}$ during the deposition. Volume ratio of gases in the reaction chamber was $\left[\mathrm{B}_{2} \mathrm{H}_{6}\right] /\left[\mathrm{SiH}_{4}\right]=10^{-4}$.

Yb:KGW laser system with repetition rate of $200 \mathrm{kHz}$ and emission wavelength of $515 \mathrm{~nm}$ was used for a-Si:H films processing. Pulse duration was $330 \mathrm{fs}$. Laser spot had a form of an ellipse with semiaxis of 8 and $50 \mu \mathrm{m}$. We used the scanning method of processing. Scanning rate was $5 \mathrm{~mm} / \mathrm{s}$. Adjacent 'scanning stripe' was shifted by a quarter of the laser spot width with respect to the preceding one. This overlap was used to increase uniformity of produced modification. Modification of a-Si:H structure was carried out with different laser fluences: 0, 16, 24, 48 and $64 \mathrm{~mJ} / \mathrm{cm}^{2}$.

For electrical and photoelectric measurements aluminum contacts were evaporated on film surfaces parallel to the direction of laser scanning. Distance between contacts and length of contacts were 0,5 and 4 $\mathrm{mm}$ correspondingly. Studied samples were placed in optical cryostat that allows to carry out measurements in the range of temperatures from 100 to $480 \mathrm{~K}$. All measurements were carried out in vacuum with residual pressure of $10^{-3} \mathrm{~Pa}$ after annealing of the films at the temperature of $160{ }^{\circ} \mathrm{C}$ within 5 minutes.

Information about the films' structure was obtained from analysis of Raman spectra that were measured by micro-Raman spectrometer Horiba Jobin Yvon HR800 during samples excitation with radiation with wavelength equal to $488 \mathrm{~nm}$.

To estimate the crystalline phase volume fraction in the studied modified silicon films the decomposition of Raman spectra onto phonon modes was conducted. It is known that three main maxima can be distinguished in Raman spectra of two-phase hydrogenated silicon, namely, maxima centered near frequencies of $\omega_{a}=480 \mathrm{~cm}^{-1}, \omega_{c}=520 \mathrm{~cm}^{-1}$ and $\omega_{i}=500 \mathrm{~cm}^{-1}$ [3]. Maximum near the frequency of $\omega_{a}=480 \mathrm{~cm}^{-1}$ corre-

\footnotetext{
*forsh@phys.msu.ru
} 
sponds to transverse optical (TO) phonon mode in amorphous silicon structure, while the maximum at $\omega_{c}=520 \mathrm{~cm}^{-1}$ corresponds to TO phonons of crystalline silicon. Maximum near the frequency of $\omega_{i}=500 \mathrm{~cm}^{-1}$ is called intermediate. This peak is due to the intermediate phase at the interface between $\mathrm{Si}$ nanocrystals and amorphous silicon matrix. To estimate a volume fraction of the crystalline phase $f_{c}$ in the investigated a-Si:H films that were exposed to laser crystallization, integrated intensities $I_{a}, I_{i}$ and $I_{c}$ of TO phonon modes with maxima near frequencies of $\omega_{a}, \omega_{i}$ and $\omega_{c}$ accordingly that contribute to the measured Raman spectra were calculated. The value of $f_{c}$ was determined from the following expression:

$$
f_{c}=\frac{I_{c}+I_{i}}{\sigma_{0} I_{a}+I_{c}+I_{i}}
$$

where $\sigma_{0}=0.1+\exp \left(-d_{n c-S i} / 25\right)-$ empirical relation for integral cross-section ratios of Raman light scattering in crystalline and amorphous phases of silicon [4], $d_{n c-S i}$ - average diameter of silicon nanocrystals in nanometers.

\section{RESULTS AND DISCUSSION}

Raman spectra of studied films are shown in Figure 1. These spectra were obtained for a-Si:H films that were treated with laser radiation with different fluences. The spectra were taken from the side of the film that was exposed to radiation during laser processing.

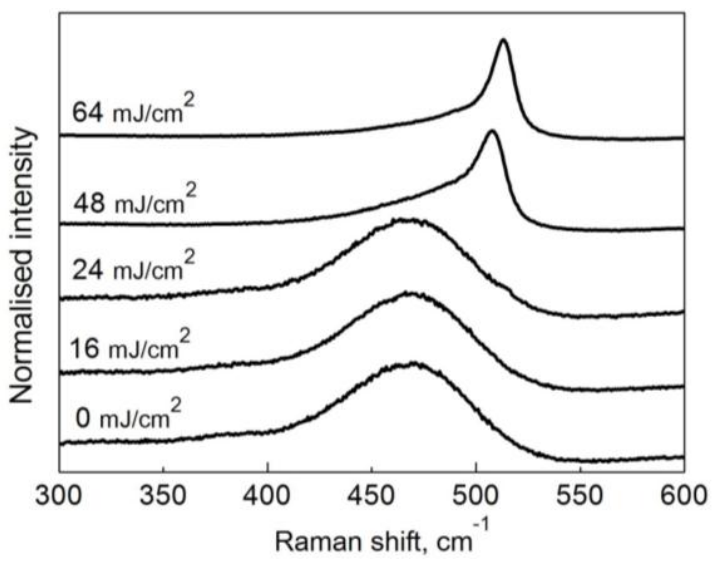

Fig. 1 - Raman spectra of boron doped a-Si:H films treated with different laser fluences, which are shown in the figure

As can be seen from Fig. 1, the lineshape of Raman spectrum changes from "amorphous" to "nanocrystalline" with increase of laser fluence used for the treatment. The values of the crystalline phase volume fraction in the films modified by laser radiation were obtained from analysis of the measured Raman spectra. Figure 2 shows variation of calculated crystalline phase volume fraction $f_{c}$ with increase of laser fluence. The comparison of the obtained data with similar studies of laser-crystallized intrinsic (undoped) a-Si:H films shows that boron doping decreases crystallization threshold, i.e. less energies are needed to start the crystallization process.

Modification of films' structure following the laser crystallization leads to modification of their electrical

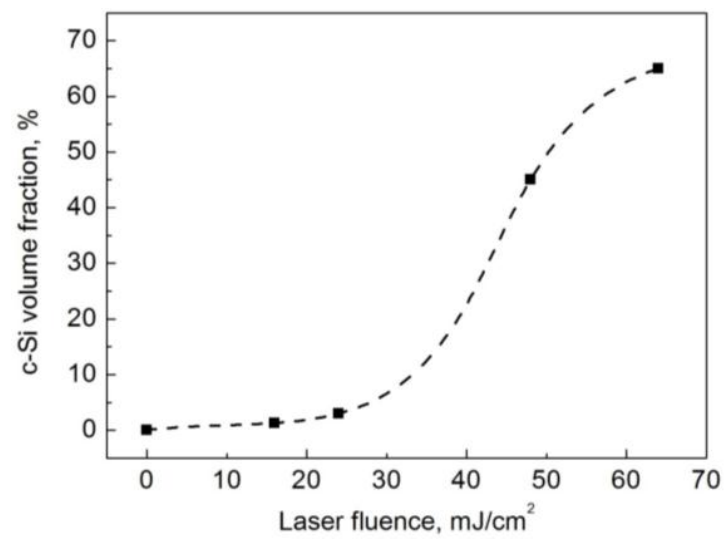

Fig. 2 - Crystalline volume fraction in studied films against laser fluence

properties. Figure 3 shows temperature dependences of conductivity of boron doped a-Si:H films which were exposed to laser irradiation with different intensities. Temperature dependences of conductivity of undoped a$\mathrm{Si}: \mathrm{H}$ and of doped a-Si:H film before laser treatment are presented in the same figure for comparison. As illustrated in Fig. 3, the conductivity of boron doped a$\mathrm{Si}: \mathrm{H}$ film at room temperature is three orders larger than the conductivity of undoped a-Si:H. Herewith the activation energies of temperature dependences of dark conductivity $\left(E_{a}\right)$ for undoped and doped films are 0.97 $\mathrm{eV}$ and $0.52 \mathrm{eV}$ respectively. It should be noted that laser-modified films have a double-layer structure. The values of conductivity from Fig. 3 are the values of effective conductivity, in which the layers of nanocrystalline silicon play a key part. As follow from Fig. 3, a larger intensity of laser irradiation that is used for processing of a-Si:H films forms a film with larger conductivity and smaller activation energy.

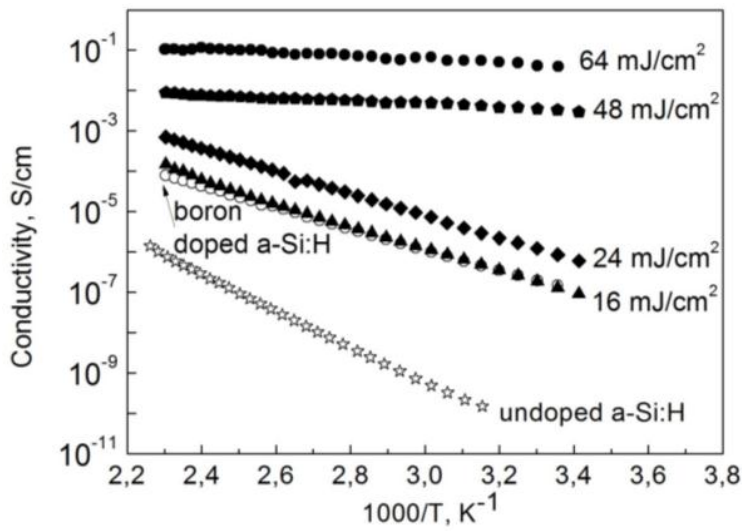

Fig. 3 - Temperature dependences of dark conductivity of a$\mathrm{Si}: \mathrm{H}$ films that were irradiated by femtosecond laser pulses with different fluences specified in the figure

The effect of laser fluence on room temperature conductivity and activation energy of the modified films is shown in the Figure 4. Analysis of the obtained dependences shows that no significant changes of conductivity and activation energy are observed for the films that were treated with laser fluences lower than $24 \mathrm{~mJ} / \mathrm{cm}^{2}$. The values of conductivity are in the range from $3 \cdot 10^{-7}$ to $3 \cdot 10^{-6} \mathrm{~S} / \mathrm{cm}$, while their activation energies values lie within $0.50-0.56 \mathrm{eV}$ at room tempera- 
ture. At the same time, a sharp increase of conductivity is observed for the films that were laser irradiated with laser fluences of 48 and $64 \mathrm{~mJ} / \mathrm{cm}^{2}$; conductivity values at room temperature are equal to $4 \cdot 10^{-3} \mathrm{~S} / \mathrm{cm}$ and $5 \cdot 10^{-2} \mathrm{~S} / \mathrm{cm}$ respectively. According to Fig. 2 and Fig. 4, observed sharp increase in conductivity correlates with sharp increase in the crystalline phase fraction in film's structure. Thus, one of the factors that leads to the increase of film's conductivity is its crystallization. Noteworthy, that the activation energy for film with the highest crystalline content is only $0,08 \mathrm{eV}$, which is the minimal obtained value. Let us remark here that activation energy of temperature dependence of dark conductivity of undoped nc-Si:H films was previously found to be in the range of $0.4-0.6 \mathrm{eV}$ [5]. Therefore remarkably small value of activation energy that was obtained for laser-modified lightly boron doped a$\mathrm{Si}: \mathrm{H}$ film points to the formation of heavily boron doped nanocrystalline silicon films. It could be due to essentially larger doping efficiency of nc-Si:H as compared to the doping efficiency of amorphous silicon.

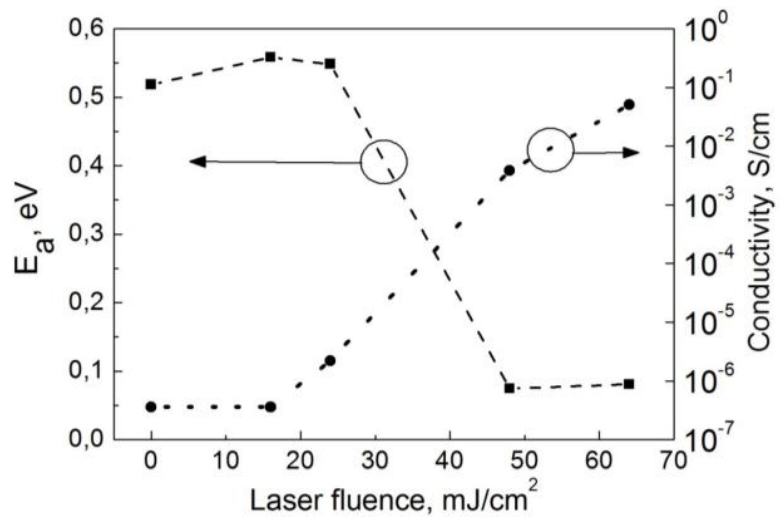

Fig. 4 - Dependence of the activation energy and conductivity of the modified films at room temperature on laser fluence used for their treatment

Spectral dependences of absorption coefficient $(\alpha)$ of laser-modified boron doped a-Si:H films were measured by constant photocurrent method [6]. Figure 5 shows obtained dependences for films irradiated by laser pulses with fluences in the range of $0-24 \mathrm{~mJ} / \mathrm{cm}^{2}$. Films formed upon laser irradiation of a-Si:H films with fluences of 48 and $64 \mathrm{~mJ} / \mathrm{cm}^{2}$ possess larger dark conductivity and lower photosensitivity. It restricts the use of constant photocurrent method for studying these films.

Films of a-Si:H that were processed by femtosecond laser radiation with fluences up to $24 \mathrm{~mJ} / \mathrm{cm}^{2}$ have absorption coefficient spectrum that is characteristic for

\section{REFERENCES}

1. K. Yamamoto, A. Nakajima, M. Yoshimi, T. Sawada, S. Fukuda, T. Suezaki, M. Ichikawa, Y. Koi, M. Goto, T. Meguro, T. Matsuda, M. Kondo, T. Sasaki, Y. Tawada., J. Sol. Energ. 77, 939 (2004).

2. M. Khenkin, A. Emelyanov, P. Forsh, A. Kazanskii, M. Beresna, M. Gecevicius, P. Kazansky, Can. J. Phys. 92 883 (2014). boron doped a-Si:H films. Since the constant photocurrent method only registers absorption that leads to photoconductivity, obtained result points out that photoconductivity in the samples is defined by amorphous phase. At the same time, the increase of laser fluence leads to the increase of absorption in range of light quanta energies from $1 \mathrm{eV}$ to $1.5 \mathrm{eV}$. It points to the increase of defect concentration in these samples.

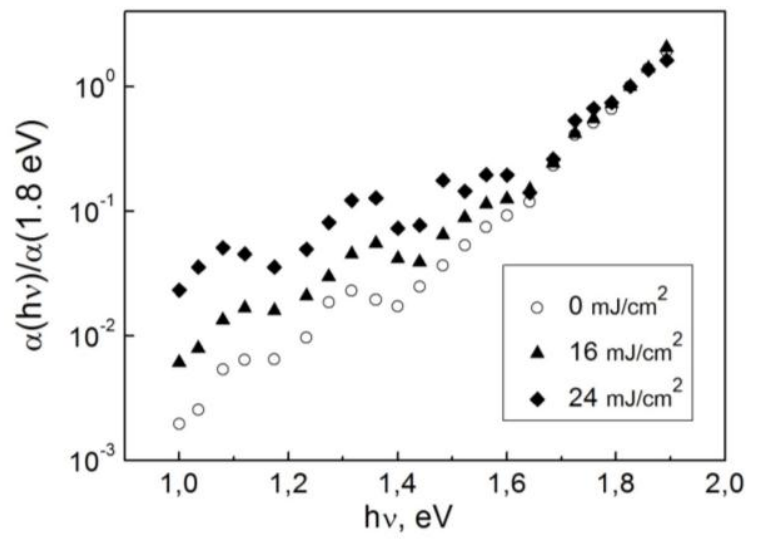

Fig. 5 - Spectral dependences of absorption coefficient measured by constant photocurrent method for the samples processed by laser radiation. Laser fluences are specified in the figure

\section{CONCLUSION}

In summary, the influence of femtosecond laser irradiation on structure and conductivity of lightly boron doped a-Si:H films was studied. Laser treatment with fluence exceeding $24 \mathrm{~mJ} / \mathrm{cm}^{2}$ resulted in the partial crystallization of a-Si:H with formation of nanocrystalline silicon layer on film surface. Modification of films' structure resulted in 5 orders of magnitude increase of their conductivity value and considerable decrease of activation energy of temperature dependence of dark conductivity. Small value of activation energy of modified films points to the formation of heavily boron doped nanocrystalline silicon that could be explained by the increase of doping efficiency of the films during their crystallization.

\section{ACKNOWLEDGEMENT}

Authors are grateful to Dr. O.I. Konkov for boron doped a-Si:H films and to A.A. Khomich for measurements of Raman spectra.

This work was supported by the Ministry of Education and Science of the Russian Federation (grant no. 14.604.21.0085, identification number RFMEFI60414X0085).

3. N.M. Liao, W. Li, Y.D. Jiang, Y.J. Kuang, K.C. Qi, Z.M. Wu, S.B. Li, J. Appl. Phys. A 91, 349 (2008).

4. V.G. Golubev, V.Yu. Davydov, A.V. Medvedev, A.B. Pevtsov, Phys. Solid State 39, 1197 (1997)

5. P. Alpuim, V. Chu, J. Vac. Sci. Technol. A 19, 2328 (2001).

6. M. Vaněček, J. Kočka, J. Stuchlik, Z. Kožišek, O. Štika, A. Třiska, J. Sol. Energ. Mater. 8, 411 (1983). 